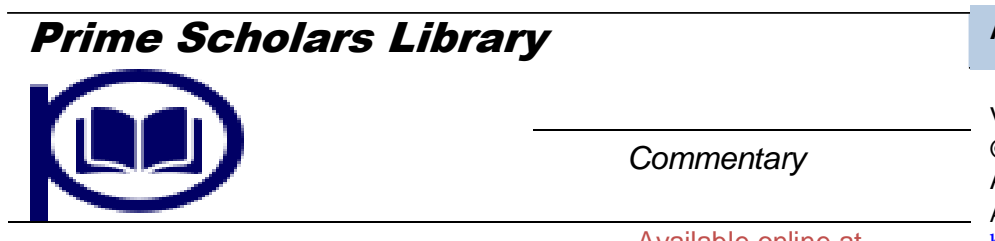

Available online at

https://primescholarslibrary.org/

\title{
A note on cytopathic effect
}

\author{
Paula Tim* \\ Department of Microbiology, University of Washington, Washington, USA.
}

\section{DESCRIPTION}

Cytopathic Effect (CPE) refers to the structural changes of host cells caused by virus invasion. Infection with the virus causes the host cell to lyse, or when the cell dies because it cannot replicate. Both of these effects occur due to CPE. If the virus causes these morphological changes in the host cell, it is said to be cytopathic. Common examples of CPE include rounding of infected cells, fusion with neighboring cells to form syncytia, and the appearance of nuclear or cytoplasmic inclusions. EPC and other changes in cell morphology are just a few of the many effects of cytocidal viruses. When a cytocidal virus infects a permissive cell, the virus kills the host cell through changes in cell morphology, cell physiology, and subsequent biosynthetic events. These changes are necessary for efficient virus replication, but at the expense of the host cell. ECP is an important aspect of virus infection in diagnosis. Under the low magnification of a light microscope, many CPEs can be seen in unfixed and unstained cells, with the condenser down and the iris diaphragm partially closed. However, for some CPEs, that is, inclusion bodies, the cells must be fixed and stained and then viewed under a light microscope.

The CPE of some viruses is characteristic, so it can be an important tool for virologists to diagnose infected animals or humans. The incidence of CPE is also an important characteristic that virologists can use to identify virus types. If CPE occurs after 4 to 5 days outside the body and the multiplicity of infections is low, the virus is considered to be slow. If CPE appears with a low multiplicity of infection after 1 to 2 days outside the body, the virus is considered to spread rapidly. Vaccination always occurs with a low multiplicity of infections, because with a high multiplicity of infections all CPEs will occur rapidly.
The first sign of viral infection is rounding of cells. Inclusion bodies often appear in the nucleus and cytoplasm of host cells. Inclusion bodies can first be identified from a patient's blood smear or stained section of infected tissue through an optical microscope. However, in order to fully characterize its composition, electron microscopy must be performed. Inclusion bodies can be the accumulation of by-products of virus replication or altered host cell structures or organelles.

Some viral infections can cause a rare type of CPE, the formation of syncytia. The syncytia are a large cytoplasmic mass that contains many nuclei. They are usually produced by the fusion of infected cells. This mechanism is useful for viruses because it allows the virus to spread from infected to uninfected cells. Viral infection may have a clinically relevant CPE phenotype. For example, for the Hepatitis C Virus (HCV), hepatic steatosis is a sufficient characteristic of the virus to help identify the genotype, that is, the genetic makeup of the virus. Patients with HCV genotype 3 are more likely to develop hepatic steatosis than patients with genotype 1 . In addition, CPE can be used during the study to determine the efficacy of new drugs. A test method to detect dengue virus CPE has been developed to assess cell viability.

Due to the host cell specificity of CPE, researchers can also use them to test for differences in experiments. For many viral infections, different host cell strains can have characteristic responses. Today, the research community has many concerns about the efficacy and purity of cell lines. 